\title{
433 - Burden of Disease Associated with Dementia-related Psychosis and Dementia-related Agitation \& Aggression Using a National Long-term Care US Database
}

Author list: Nazia Rashid ${ }^{1}$, Sherry Andes ${ }^{1}$, Vic Abler ${ }^{1}$, Leslie Citrome ${ }^{2}$

${ }^{1}$ ACADIA Pharmaceuticals Inc., San Diego, CA, USA

${ }^{2}$ New York Medical College, Valhalla, NY, USA

\begin{abstract}
:
Objective: Compare burden of disease among patients with dementia-related psychosis (DRP), dementia without psychosis (dementia only), and dementia-related agitation/aggression (DAA) in long-term care (LTC) facilities.
\end{abstract}

Background: Patients with dementia often experience neuropsychiatric symptoms (NPS), including psychosis and agitation/aggression. Real-world data on the comorbidity profile of DRP and DAA patients are limited.

Design/Methods: Dementia patients were identified from a US LTC database based on $\geq 2$ dementia diagnosis codes or 1 dementia diagnosis code and antidementia therapy prescription during 1 Jan 2013 to 30 May 2017. Patients were categorized into DRP ( $\geq 2$ psychosis or 1 psychosis diagnosis code and prescription of antipsychotic therapy and no history of agitation/aggression diagnosis), dementia only (no psychosis or agitation/aggression diagnosis and no history of antipsychotic therapy [dementia only]), and DAA ( $\geq 2$ diagnosis codes of agitation/aggression and no history of psychosis diagnosis or antipsychotic therapy) groups (index date). Comorbidities and concomitant therapies were defined during 12 months prior to index date.

Results: There were 26,002 dementia residents: DRP ( $n=11,921 ; 46 \%)$; dementia only ( $n=11,432 ; 44 \%)$; DAA ( $n=2649 ; 10 \%)$. DRP patients were younger (mean age 80.8 years) than dementia only ( 84.3 years) or DAA ( 83.8 years). DRP patients were sicker overall versus dementia only: anemia ( $32 \%$ vs $29 \%$ ); anxiety ( $55 \%$ vs $33 \%$ ); bladder disorders ( $19 \%$ vs $13 \%$ ); depression ( $75 \%$ vs $58 \%$ ); hypertension ( $43 \%$ vs $33 \%$ ); diabetes ( $43 \%$ vs $38 \%$ ); insomnia disorders ( $32 \%$ vs $19 \%$ ); (all $P<0.05$ ). More DAA patients had anxiety (43\%), depression (66\%), hypertension (43\%), and insomnia disorders (26\%) than dementia only (all $P<0.05)$. Most DRP patients $(94.3 \%$ ) received off-label treatment for DRP; approximately one third (31.6\%) of DAA patients received off-label treatment for DRP.

Conclusions: This study, the first of its type to use a US LTC database, demonstrated a significant comorbidity burden associated with DRP or DAA compared with dementia only, which should be considered when using off-label treatments. These data highlight the need for safe and effective treatments for dementia NPS.

Study Sponsored By: ACADIA Pharmaceuticals Inc.

\section{Disclosures}

NR, SA, VA are employees of ACADIA Pharmaceuticals

LC has served as a consultant, speaker, holds stock in, or receives royalties from: Acadia, Alkermes, Allergan, Avanir, BioXcel, Eisai, Impel, Indivior, Intra-Cellular Therapies, Janssen, Lundbeck, Luye, Merck, Neurocrine, Noven, Osmotica, Otsuka, Pfizer, Sage, Shire, Sunovion, Takeda, Teva, Vanda, Bristol-Myers Squibb, Eli Lilly, J \& J; Wiley (Editor-in-Chief, International Journal of Clinical Practice), UpToDate (reviewer), Springer Healthcare (book) 
This submission is an encore of a poster abstract originally presented at ISPOR 2019, New Orleans, LA, USA, May 18-22, 2019; original presentation under the same title. 